\title{
ORMDL3 is a differentially expressed gene in brain metastatic human breast cancer.
}

Shahan Mamoor, MS ${ }^{1}$

${ }^{1}$ shahanmamoor@gmail.com

Metastasis to the brain is a clinical problem in patients with breast cancer ${ }^{1-3}$. We mined published microarray data ${ }^{4,5}$ to compare primary and metastatic tumor transcriptomes for the discovery of genes associated with brain metastasis in humans with metastatic breast cancer. We found that ORMDL sphingolipid biosynthesis regulator 3, encoded by ORMDL3, was among the genes whose expression was most different in the brain metastases of patients with metastatic breast cancer as compared to primary tumors of the breast. ORMDL3 mRNA was present at increased quantities in brain metastatic tissues as compared to primary tumors of the breast. Importantly, expression of ORMDL3 in primary tumors was significantly correlated with patient overall survival. Modulation of ORMDL3 expression may be relevant to the biology by which tumor cells metastasize from the breast to the brain in humans with metastatic breast cancer.

Keywords: breast cancer, metastasis, brain metastases, central nervous system metastases, ORMDL sphingolipid biosynthesis regulator 3, ORMDL3, systems biology of breast cancer, targeted therapeutics in breast cancer. 
One report described a $34 \%$ incidence of central nervous system metastases in patients treated with trastuzumab for breast cancer ${ }^{2}$. More recently, the NEfERT-T clinical trial ${ }^{6}$ which compared administration of either neratinib or trastuzumab in conjunction with paclitaxel demonstrated that in a randomized, controlled setting, in breast cancer patients treated with neratinib, not only was the incidence of central nervous system recurrence significantly lower, the time to central nervous system metastasis was significantly delayed as compared to patients administered trastuzumab ${ }^{6}$. The alarmingly high rate of central nervous system metastasis described, as well as data, both anecdotal ${ }^{2}$ and from a randomized, controlled setting ${ }^{6}$ illustrating that treatment with trastuzumab may be associated with these events demands an enhanced understanding of the transcriptional makeup of brain metastatic tissues to support identification of therapeutic targets, whether they are treatment-related or not. We performed a global comparative analysis of primary and metastatic tumors in patients with brain metastatic breast cancer ${ }^{4,5}$. We discovered differential and increased expression of the gene encoding ORMDL sphingolipid biosynthesis regulator 3, ORMDL3, in brain metastatic tissues of patients with metastatic breast cancer.

\section{Methods}

We used datasets GSE10893 ${ }^{4}$ and GSE72653 5 for this global differential gene expression analysis of brain metastatic breast cancer in conjunction with GEO2R. GSE10893 was generated using Agilent-011521 Human 1A Microarray G4110A technology with $n=11$ primary breast tumors and $n=3$ brain metastases from patients with breast cancer; analysis was performed using platform GPL885. GSE72653 was generated using Agilent-014850 Whole Human Genome Microarray $4 \times 44 \mathrm{~K}$ G4 $112 \mathrm{~F}$ with $n=9$ normal epithelium and $n=9$ tumors of the breast from patients with breast cancer; analysis was performed using platform GPL6480. The Benjamini and Hochberg method of $p$-value adjustment was used for ranking of differential expression but raw $p$-values were used to assess statistical significance of global differential expression. Log-transformation of data was auto-detected, and the NCBI generated category of platform annotation was used. A statistical test was performed to evaluate whether ORMDL3 gene expression was significantly different between primary tumors of the breast and brain metastases in humans with breast cancer using a two-tailed t-test. For Kaplan-Meier survival analysis, we used the Kaplan-Meier plotter online tool ${ }^{7}$ for correlation of ORMDL3 mRNA expression levels with overall survival in $n=626$ breast cancer patients.

\section{$\underline{\text { Results }}$}

We performed global comparative transcriptome analysis of primary and metastatic tumor tissues of patients with breast cancer and metastatic breast cancer using published microarray data $^{4,5}$ to describe the transcriptional landscape of brain metastasis in human breast cancer in an unbiased fashion and for the discovery of novel therapeutic targets.

\section{ORMDL3 is differentially expressed in the brain metastases of patients with brain metastatic breast cancer.}

Through blind, systems-level analysis of published microarray data ${ }^{4}$, we identified ORMDL sphingolipid biosynthesis regulator 3, encoded by ORMDL3, as a differentially expressed gene in the breast metastatic tissues of humans with breast cancer (Table 1). When sorting each of the genes expressed in brain metastases based on significance of difference as 
compared to primary tumors of the breast in patients with breast cancer, ORMDL3 ranked 217 out of 17418 total transcripts (Chart 1), equating to $98.8 \%$ differential expression. Differential expression of ORMDL3 in the brain metastases of patients with metastatic breast cancer was statistically significant (Chart $1 ; p=6.14 \mathrm{E}-04)$.

To attempt to validate differential transcriptome-wide differential expression of ORMDL3 in human breast cancer, we queried a second microarray dataset ${ }^{5}$, here comparing normal breast tissues and primary tumors of the breast. Again, we identified ORMDL3 as a differentially expressed gene in the tumor tissues of patients with breast cancer (Chart 2). When sorting each of the genes expressed in primary tumors of the breast based on significance of difference as compared to normal breast tissues, ORMDL3 ranked 8706 out of 41093 total transcripts (Chart 2), equating to $78.8 \%$ differential expression. Differential expression of ORMDL3 in the primary tumors of patients with breast cancer was statistically significant (Chart $2 ; p=3.61 \mathrm{E}-02$ ). Thus, differential expression of ORMDL3, transcriptome-wide, in the primary tumors of women with breast cancer was conserved across two independent microarray datasets, both in primary and metastatic tumor tissues.

ORMDL3 is expressed at higher levels in the brain metastases of patients with metastatic breast cancer.

We obtained exact mRNA expression levels for ORMDL3, in primary tumors of the breast and in brain metastasis of patients with brain metastatic breast cancer to determine direction and statistical significance of change in ORMDL3 expression in brain metastatic tissues. We observed significantly higher expression of ORMDL3 in the brain metastases of patients with breast cancer as compared to primary tumors of the breast: ORMDL3 was expressed at $0.63 \pm$ 0.36 arbitrary units (AU) in primary tumors of the breast, while it was expressed at $2.14 \pm 1.15$ AU in brain metastatic tissues (Figure 1). The difference in ORMDL3 mRNA levels between primary tumors of the breast and brain metastatic tissues was statistically significant (Figure 1; $p=0.001403)$.

\section{ORMDL3 expression is significantly correlated with survival outcomes in human breast} cancer.

We performed Kaplan-Meier survival analysis ${ }^{8}$ in $n=626$ breast cancer patients in total, to evaluate whether ORMDL3 tumor expression was correlated with survival outcomes in breast cancer. We observed a statistically significant correlation between primary tumor expression of ORMDL3 and overall survival (DMFS) in patients with breast cancer (Figure 2). Patients whose primary tumors expressed low levels of ORMDL3 possessed median DMFS of 63.96 months, while patients whose tumors expressed high levels of ORMDL3 possessed median RFS of 110.4 months. This difference in DMFS based on ORMDL3 tumor expression in patients with breast cancer was statistically significant (Figure 2, Chart 3; logrank $p$-value: 0.036; hazard ratio: 0.72 (0.52-0.98).

Thus, by mining published microarray data ${ }^{4,5}$ in an unbiased and systematic fashion, we identified ORMDL sphingolipid biosynthesis regulator 3, encoded by ORMDL3, as among the genes whose expression was most different, transcriptome-wide, in the brain metastases of 
patients with breast cancer, both when compared to primary tumors of the breast and to normal breast tissues; we observed significantly increased expression of ORMDL 3 in brain metastases as compared to primary tumors of the breast. Further, we found a significant correlation between ORMDL3 expression and patient survival outcomes, as overall survival was significantly greater in patients whose primary tumors expressed higher levels of ORMDL3 as compared to patients whose primary tumors expressed lower levels of ORMDL3.

\section{Discussion}

We provided evidence here that ORMDL sphingolipid biosynthesis regulator 3, encoded by ORMDL3, is among the genes whose expression is most different in the brain metastases and primary tumors of humans with breast cancer, that ORMDL3 mRNA is present at significantly increased quantities in brain metastatic tissues as compared to primary tumors of the breast, and that primary tumor ORMDL3 expression is significantly correlated with patient survival outcomes in human breast cancer. Evaluation of the effects of genetic depletion of ORMDL3 in mouse models of metastatic breast cancer on metastasis to the central nervous system is merited. Modulation of ORMDL3 expression may be relevant to the processes by which breast cancer cells exit the breast, enter the vasculature and/or lymphatics, reside in the lymph nodes, evade immune clearance, breach the blood-brain barrier and colonize the brain. 


\section{References}

1. Lin, N.U., Amiri-Kordestani, L., Palmieri, D., Liewehr, D.J. and Steeg, P.S., 2013. CNS metastases in breast cancer: old challenge, new frontiers.

2. Bendell, J.C., Domchek, S.M., Burstein, H.J., Harris, L., Younger, J., Kuter, I., Bunnell, C., Rue, M., Gelman, R. and Winer, E., 2003. Central nervous system metastases in women who receive trastuzumab-based therapy for metastatic breast carcinoma. Cancer, 97(12), pp.2972-2977.

3. Tsukada, Y., Fouad, A., Pickren, J.W. and Lane, W.W., 1983. Central nervous system metastasis from breast carcinoma autopsy study. Cancer, 52(12), pp.2349-2354.

4. Weigman, V.J., Chao, H.H., Shabalin, A.A., He, X., Parker, J.S., Nordgard, S.H., Grushko, T., Huo, D., Nwachukwu, C., Nobel, A. and Kristensen, V.N., 2012. Basal-like Breast cancer DNA copy number losses identify genes involved in genomic instability, response to therapy, and patient survival. Breast cancer research and treatment, 133(3), pp.865-880.

5. Abdalla, M., Tran-Thanh, D., Moreno, J., Iakovlev, V., Nair, R., Kanwar, N., Abdalla, M., Lee, J.P., Kwan, J.Y.Y., Cawthorn, T.R. and Warren, K., 2017. Mapping genomic and transcriptomic alterations spatially in epithelial cells adjacent to human breast carcinoma. Nature communications, 8(1), pp.1-11.

6. Awada, A., Colomer, R., Inoue, K., Bondarenko, I., Badwe, R.A., Demetriou, G., Lee, S.C., Mehta, A.O., Kim, S.B., Bachelot, T. and Goswami, C., 2016. Neratinib plus paclitaxel vs trastuzumab plus paclitaxel in previously untreated metastatic ERBB2-positive breast cancer: the NEfERT-T randomized clinical trial. JAMA oncology, 2(12), pp.1557-1564.

7. Györffy, B., Lanczky, A., Eklund, A.C., Denkert, C., Budczies, J., Li, Q. and Szallasi, Z., 2010. An online survival analysis tool to rapidly assess the effect of 22,277 genes on breast cancer prognosis using microarray data of 1,809 patients. Breast cancer research and treatment, 123(3), pp.725-731. 


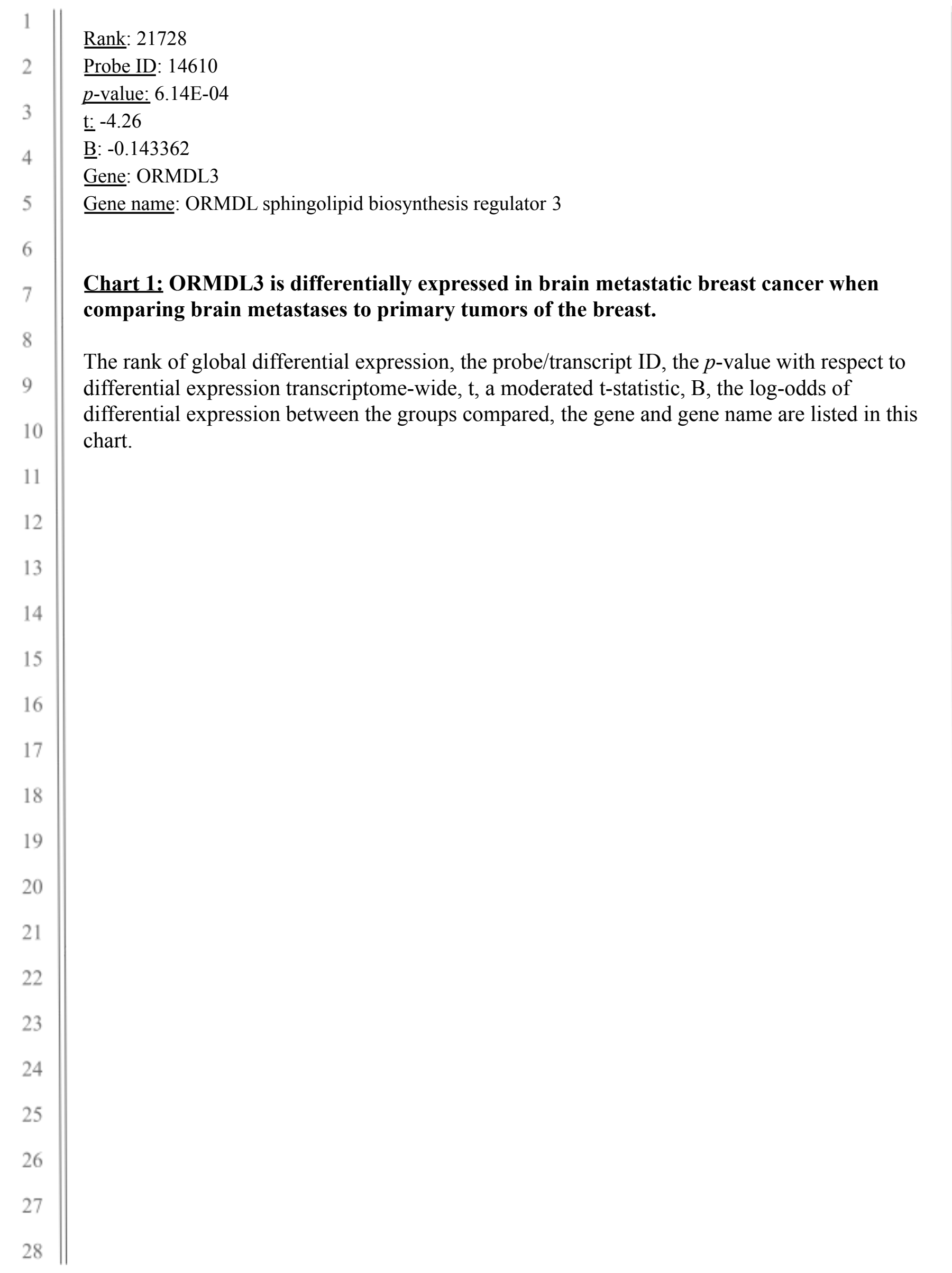




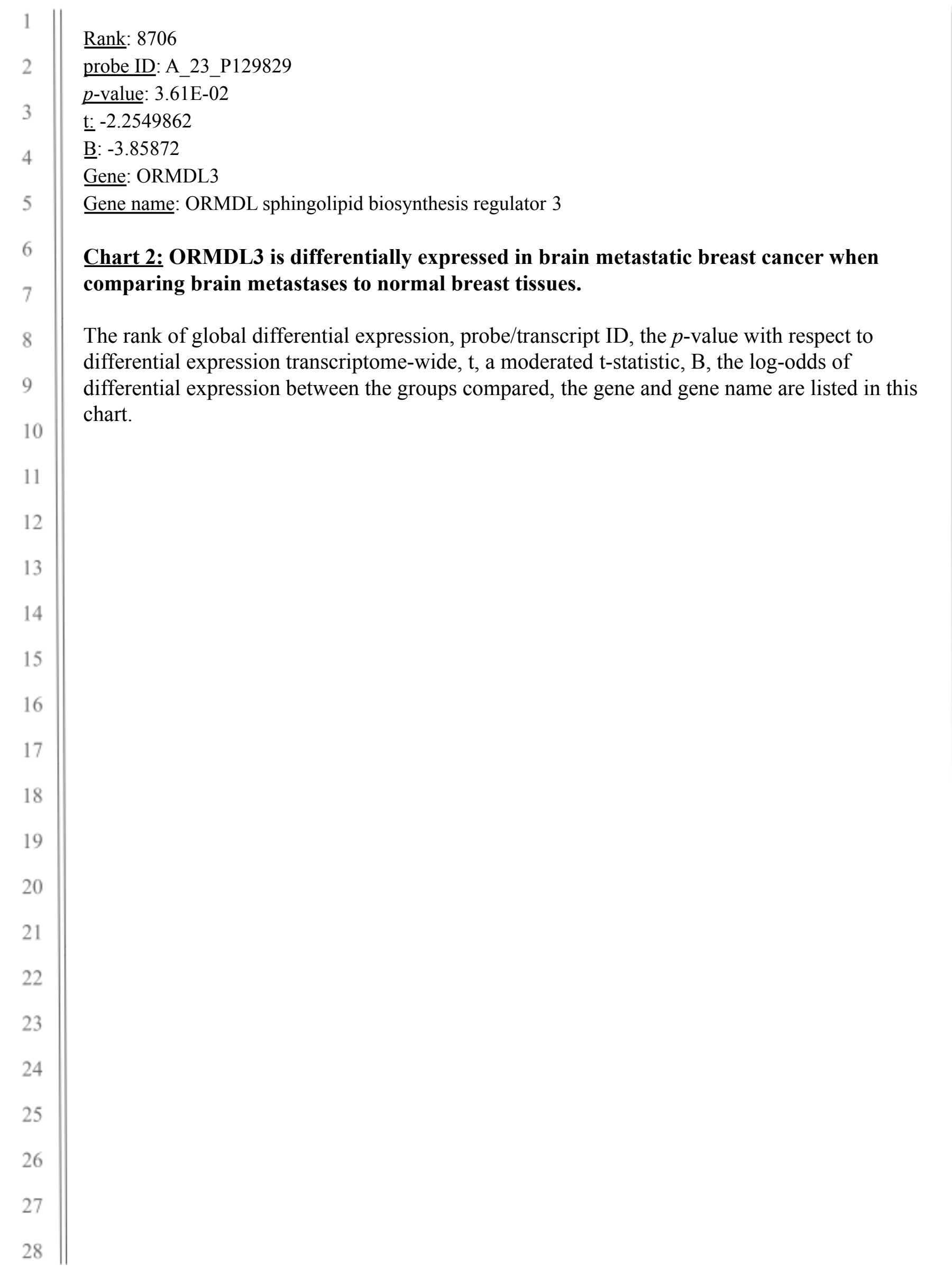




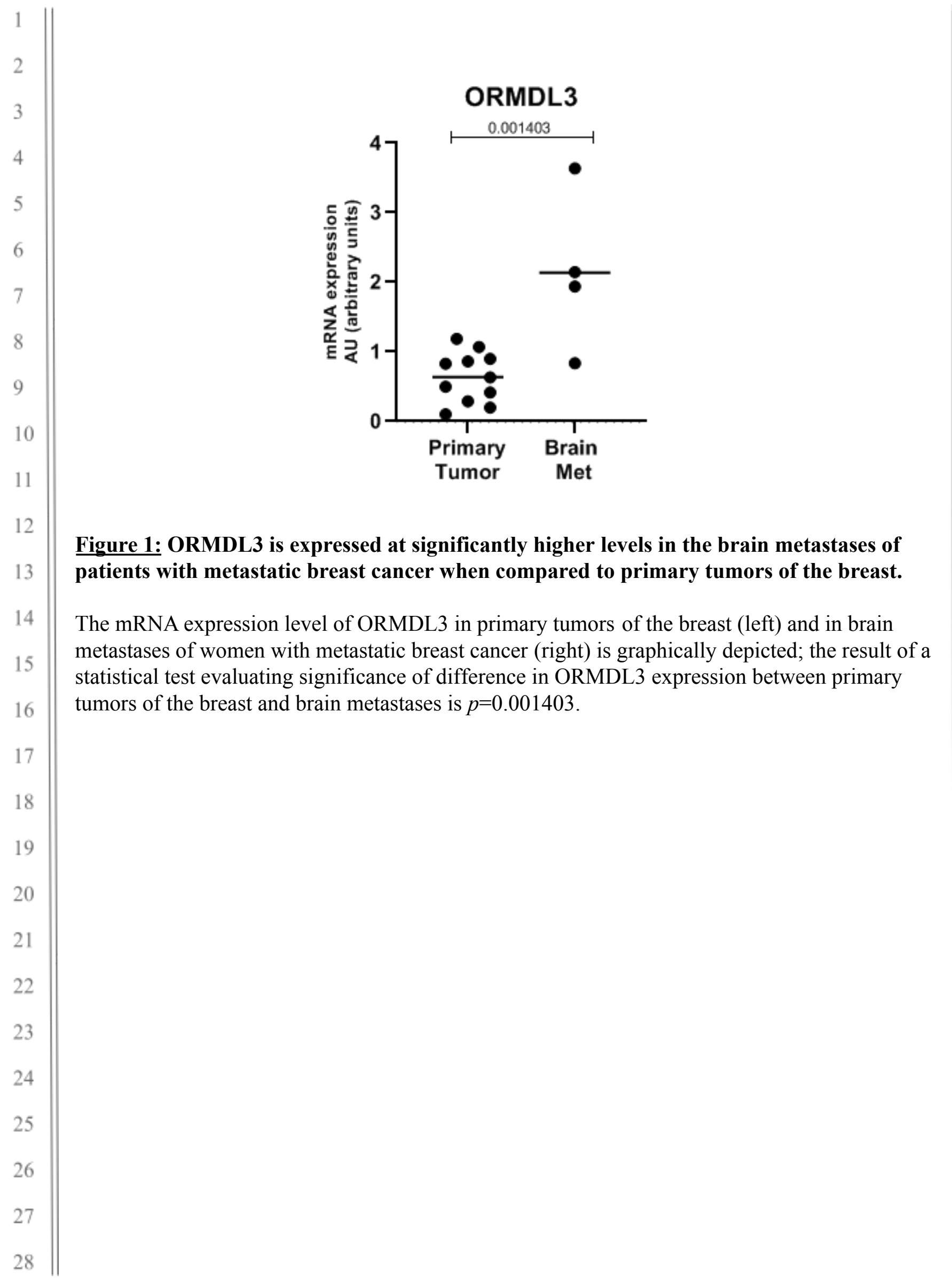




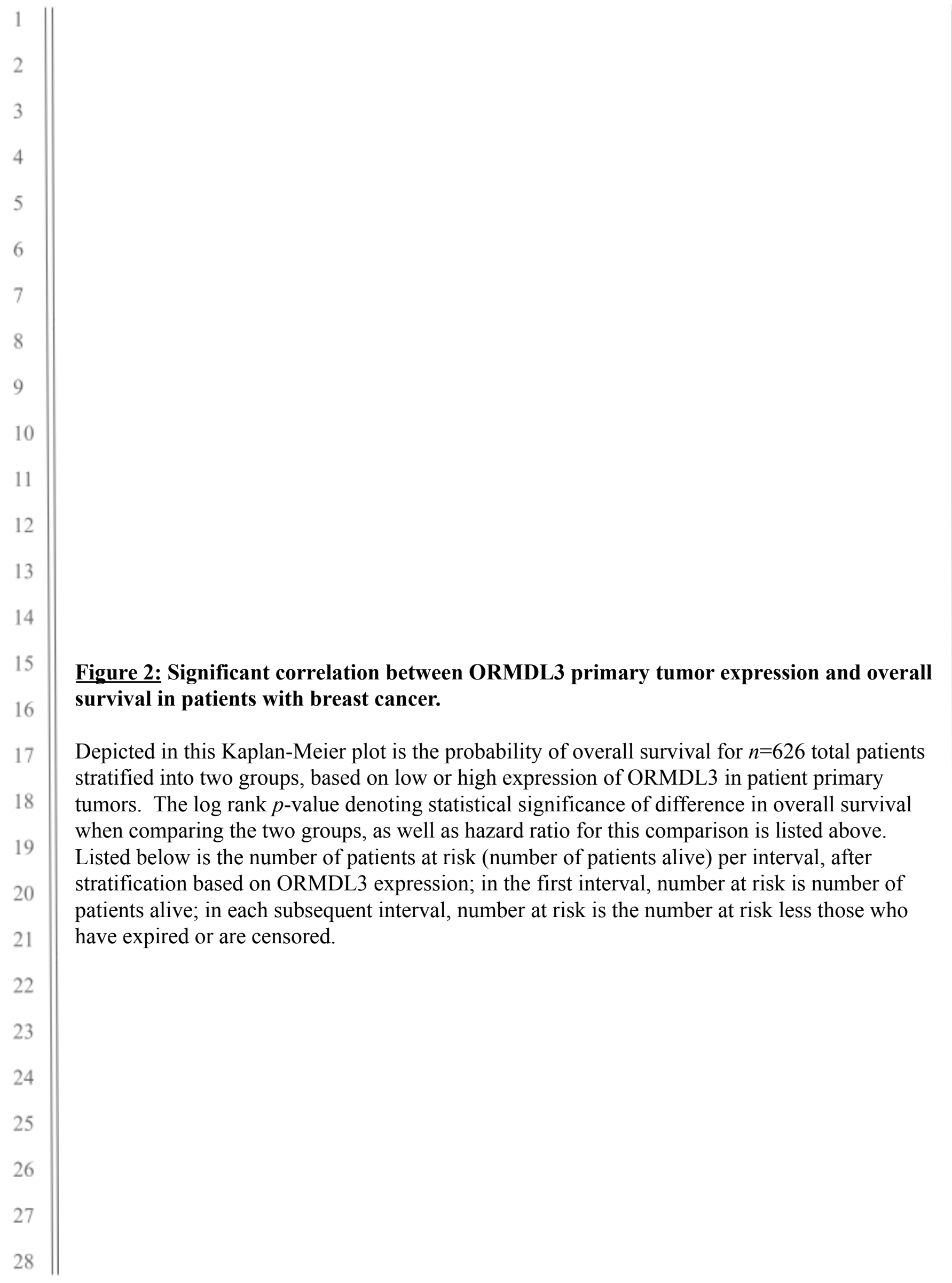

Low ORMDL3 expression: 63.96 months 
High ORMDL3 expression: 110.4 months

2

Chart 3: Median overall survival is inferior in patients with low primary tumor expression of ORMDL3.

The median OS (overall survival) of patients with low primary tumor expression of ORMDL3 and high primary tumor expression of ORMDL 3 is listed in this chart, for $n=626$ breast cancer patients. 